\title{
Influência de baixas temperaturas no desenvolvimento e aspectos bionômicos de Musca domestica (Linnaeus, 1758) (Diptera, Muscidae)
}

\author{
Luciane D'Avila Rosenthal * \\ Paulo Bretanha Ribeiro \\ Mariane D'Avila Rosenthal \\ Élvia Elena Silveira Vianna \\ Lucas Marafina Vieira Porto \\ Universidade Federal de Pelotas \\ Departamento de Microbiologia e Parasitologia, Campus Universitário - Capão do Leão \\ Caixa Postal 354, 96010-900, Pelotas - RS, Brasil \\ lulurosenthal@hotmail.com
}

Submetido em $14 / 11 / 2012$

Aceito para publicação em 23/09/02013

\section{Resumo}

Sobrevivência, longevidade, aspectos reprodutivos dos dípteros estão relacionados a fatores bióticos, abióticos e suas interações. O objetivo deste artigo consistiu em verificar a influência da estocagem de espécimes de adultos de Musca domestica sob baixas temperaturas $\left(5^{\circ} \mathrm{C}, 10^{\circ} \mathrm{C}\right)$, considerando aspectos bionômicos (sobrevivência, longevidade, número médio de ovos/fêmeas e viabilidade) de colônias pré-estabelecidas sob condições laboratoriais (temperatura $25+2^{\circ} \mathrm{C}$; UR $70+10 \%$; fotoperíodo $12 \mathrm{~h}$ ). Foram selecionados ao acaso 15 casais/repetição x 3 , acondicionados em câmaras sob temperaturas $5^{\circ} \mathrm{C}, 10^{\circ} \mathrm{C} \mathrm{e} 25^{\circ} \mathrm{C} \pm 2^{\circ} \mathrm{C}$ (controle) (Fator A). Os dípteros foram retirados das câmaras após sete, 14, 21, 28, 35 e 42 dias (Fator B), quando os sobreviventes foram quantificados e acondicionados em gaiolas, a $25^{\circ} \mathrm{C}+2^{\circ} \mathrm{C}$, até a morte do último inseto. Os resultados foram submetidos à análise estatística (Anova; Tukey $5 \%$ ). A utilização de baixas temperaturas, para estocagem de adultos, foi uma estratégia que interferiu em aspectos bionômicos. A utilização de baixas temperaturas interfere na sobrevivência de adultos, na longevidade, no número médio/ovos.fêmeas ${ }^{-1} \mathrm{e}$ na viabilidade, quando comparadas ao controle. $\mathrm{O}$ tempo de exposição de adultos em B.O.D., sob $5^{\circ} \mathrm{C}$ e $10^{\circ} \mathrm{C}$, por sete dias, permite a sobrevivência total, sobrevivência de fêmeas e machos, a longevidade, um maior número médio de ovos. fêmeas $^{-1}$ e viabilidade de ovos.

Palavras-chave: Bionomia; Condicionamento; Estocagem

\section{Abstract}

Influence of low temperatures on the development and bionomic aspects of Musca domestica (Linnaeus, 1758) (Diptera, Muscidae). Survival, longevity, and reproductive aspects of Diptera are related to biotic and abiotic factors, as well as to their interaction. The aim of this study was to determine the influence of storage of adult Musca domestica in low temperatures $\left(5^{\circ} \mathrm{C}, 10^{\circ} \mathrm{C}\right)$ by bionomic aspects (survival, longevity, 
number, and viability medium eggs.females ${ }^{-1}$ ) from pre-established colonies (temperature $25^{\circ} \mathrm{C} \pm 2{ }^{\circ} \mathrm{C}$; UR70 $\pm 10 \% ; 12 \mathrm{~h}$ photoperiod). 15 couples/repeat $\mathrm{x} 3$ were randomly selected and housed in chambers at temperatures of $5^{\circ} \mathrm{C}, 10^{\circ} \mathrm{C}$, and $25^{\circ} \mathrm{C} \pm 2^{\circ} \mathrm{C}$ (control) (Factor A). The flies were removed from the chambers at 7 , $14,21,28,35$, and 42 days (Factor B), when the survivors were counted and placed in cages at $25^{\circ} \mathrm{C} \pm 2^{\circ} \mathrm{C}$, until the death of the last fly. The results were statically tested (Anova, Tukey 5\%). The use of low temperatures for storage of adults was a strategy that interfered in bionomic aspects of the insects. The use of low temperatures interfered in adult survival, longevity, number medium/eggs.females ${ }^{-1}$, and viability when compared to control. Expose adults in B.O.D., temperature 5 and $10^{\circ} \mathrm{C}$, for seven days allows the overall survival, survival of males and females, longevity, larger number medium eggs.females ${ }^{-1}$, and egg viability.

Key words: Bionomics; Conditioning; Storage

\section{Introdução}

A interação organizada espécie/ambiente é necessária para a manutenção vital dos organismos inseridos ou distribuídos nos diversos ecossistemas, sob a interferência de inúmeros fatores (ODUM, 1988; MARTINS et al., 2010), constituindo importantes mecanismos ecológicos capazes de influenciar a dinâmica comportamental das espécies (BERRYMAN, 2002). Assim, os insetos estão sujeitos a fatores determinantes, sejam estes climáticos, ecológicos e/ou genéticos, de tal forma que possam inibir ou favorecer o desenvolvimento de uma determinada espécie, através de uma infindável complexidade de fatores que podem interferir no desenvolvimento e ciclo biológico destes (LARA, 1995).

Conforme Silveira-Neto (1976), os insetos mantém a temperatura corporal próxima a temperatura ambiente que apresenta a capacidade de exercer influência direta (desenvolvimento e comportamento) e/ou indireta (alimentação) sobre os insetos. O autor também menciona que a faixa ótima de desenvolvimento para qualquer inseto está em torno de $15^{\circ} \mathrm{C}$ a $38^{\circ} \mathrm{C}$, sendo a temperatura de $25^{\circ} \mathrm{C}$ o ponto ótimo de desenvolvimento e com maior número de descendentes.

A utilização de baixas temperaturas como forma de reduzir a taxa metabólica e o desenvolvimento tem sido a principal estratégia empregada em muitos experimentos relacionada ao armazenamento de insetos, que pode ser iniciado a partir de determinada fase de desenvolvimento, através da determinação da temperatura base (Tb) (PARRA, 2001).

De acordo com Parra (1997, 2001), conhecendo-se as exigências térmicas dos insetos, é possível realizar uma previsão ou mesmo controlar a produção em laboratório.

Segundo Rodrigues (2004), os insetos apresentam duas diferentes formas de repouso, a diapausa (interrupção do desenvolvimento) e a quinetopausa (interrupção da atividade), permitindo que possam cessar suas atividades e também o seu desenvolvimento e, durante o período mais frio, diminuir a perda de suas reservas.

Todavia, Bichão (1989) e Macedo (2001) citam que a compreensão da sazonalidade e dos fatores controladores da diapausa pode ser importante para prever o comportamento dos insetos em nível de campo ou laboratório, e permitir uma adequada manipulação dos mesmos.

Para a obtenção de sucesso em uma criação massal de insetos Parra (2001), enfatiza que é imprescindível que esta tenha continuidade durante todo o ano, havendo interrupção da mesma, não será possível em curto prazo de tempo, retornar aos níveis de uma produção massal.

Um procedimento utilizado para preservação de insetos e linhagens deverá permitir a estocagem por períodos indefinidos, e que ao mesmo tempo não causem mudanças na estrutura genotípica ou fenotípica da colônia sobre o restabelecimento após a finalização da estocagem adequada (PEGG; DIAPER, 1988).

Entretanto, de acordo com Santos (2000), o processo de regeneração ideal proporciona a recuperação da maior quantidade de insetos vivos, e a evidência inquestionável da viabilidade do material submetido ao método de conservação através de congelamento consiste na retomada do crescimento e a regeneração de um novo indivíduo. 
Díptero da família Muscidae, Musca domestica (Linnaeus, 1758) possui ampla distribuição geográfica, sendo encontrada em praticamente todos os países do mundo, mostrando-se predominante sobre os demais dípteros sinantrópicos (FERREIRA; LACERDA, 1993). Para $M$. domestica é postulado que as variações de temperatura e outros fatores climáticos influenciam diretamente sobre o tempo de duração de seu ciclo e no tamanho populacional (TORRES; OLIVEIRA; WALD, 2002).

Diante disto, como necessidade de verificar o possível procedimento de estocagem sob condições controladas, o objetivo deste trabalho consistiu em verificar a influência de baixas temperaturas controladas $\left(5^{\circ} \mathrm{C} \mathrm{e} 10^{\circ} \mathrm{C}\right.$ ) sobre aspectos bionômicos (sobrevivência e longevidade de adultos, número/médio de ovos. fêmeas $^{-1}$ e viabilidade de ovos) de Musca domestica (Linnaeus, 1758) (Diptera, Muscidae).

\section{Material e Métodos}

O experimento foi realizado no Laboratório de Biologia de Insetos, da Universidade Federal de Pelotas (UFPEL), RS, mantendo-se uma colônia de $M$. domestica pré-estabelecida às condições laboratoriais, em câmara climatizada a temperatura de $25^{\circ} \mathrm{C} \pm 2^{\circ} \mathrm{C}$, umidade relativa $70 \pm 10 \%$ e fotofase de $12 \mathrm{~h}$, cujos adultos foram capturados no Campus Universitário, Capão do Leão.

Os adultos provenientes da colônia de manutenção foram contidos em gaiolas teladas com dimensões de 30x30x30 cm (gaiola de criação) e alimentados " $a d$ libitum" com dieta composta por açúcar refinado, farinha de carne (2:1) e água, disponibilizada em copo Becker com pequenos pedaços de espuma de poliestireno sobre toda a superfície, renovada a cada dois dias.

Para ovipostura, foi fornecido aos adultos meio de cultura constituído por farinha de carne e serragem (2:1), adicionando-se água até torná-lo de consistência pastosa. Decorridas $24 \mathrm{~h}$, as posturas foram transferidas para um recipiente maior, dentro de um funil de coleta. Neste, após a eclosão, as larvas foram alimentadas até o terceiro ínstar, momento de abandono natural do substrato, caindo em um recipiente contendo serragem moderadamente úmida, para a pupariação.

As pupas foram incubadas à temperatura de $25^{\circ} \mathrm{C} \pm 2^{\circ} \mathrm{C}$, em recipientes contendo serragem moderadamente úmida, fechados com tecido de malha fina (organza) até a emergência dos adultos, para condução do experimento.

Após a emergência, os adultos foram transferidos para as gaiolas de criação e alimentados "ad libitum" com dieta e água conforme a descrição da manutenção da colônia. Decorrido o período de maturação do aparelho reprodutor, as moscas foram separadas e designadas para a etapa experimental. Foram selecionados quinze casais de adultos ao acaso, que foram individualizados para cada repetição estatística, totalizando três repetições/ tratamento a partir das colônias de manutenção, e todos os conjuntos de casais foram acondicionados em frascos de vidro contendo papel pardo ( $80 \mathrm{~g}$ ), sanfonado, fechado com organza, acrescentando-se a parte inferior do frasco dois centímetros de serragem moderadamente úmida.

Os conjuntos de casais a serem expostos as respectivas temperaturas controladas estabelecidas $\left(5^{\circ} \mathrm{C}\right.$ e $10^{\circ} \mathrm{C}$ ) foram mantidos em câmaras de desenvolvimento biológico (B.O.Ds.), com umidade relativa $70 \pm 10 \% \mathrm{e}$ fotofase de $12 \mathrm{~h}$, enquanto que os casais destinados ao tratamento controle $\left(25^{\circ} \mathrm{C} \pm 2^{\circ} \mathrm{C}\right)$ foram imediatamente mantidos nas gaiolas de criação, no laboratório, conforme descrito anteriormente no procedimento de manutenção das colônias. Com a periodicidade de sete dias, os frascos contendo os conjuntos de casais expostos as temperaturas testes, foram retirados das câmaras, procedendo-se a quantificação dos indivíduos sobreviventes, considerando-se o número de fêmeas e machos para cada repetição. Os respectivos adultos sobreviventes para cada um dos conjuntos foram acondicionados em gaiolas de criação e mantidos a temperatura ótima controlada de $25^{\circ} \mathrm{C} \pm 2^{\circ} \mathrm{C}$, conforme o tratamento controle.

Para a avaliação: a) Longevidade de adultos, diariamente procedeu-se a remoção dos indivíduos mortos das gaiolas de criação e a contagem dos indivíduos vivos até a morte do último sobrevivente, 
caracterizando a finalização experimental, para $5^{\circ} \mathrm{C}$, $10^{\circ} \mathrm{C}$ e $25^{\circ} \mathrm{C} \pm 2^{\circ} \mathrm{C}$; b) Número/médio de ovos.fêmeas ${ }^{-1}$, diariamente procedeu-se a remoção e contagem dos ovos das gaiolas de criação, obtendo-se na finalização experimental o somatório do número total de ovos, que por sua vez foi então dividido pelo número total de fêmeas sobreviventes a partir da retirada das B.O.Ds. e mantidas nas respectivas gaiolas de criação. No grupo controle procedeu-se igualmente ao grupo proveniente das B.O.Ds. no entanto este era dividido pelo número total de fêmeas sobreviventes; c) Viabilidade de ovos, foram selecionados ao acaso, diariamente, 30 ovos da massa por gaiola de criação e acondicionados em placas de Petri sobre papel filtro umedecido e mantidos em B.O.D. a $25^{\circ} \mathrm{C}+2^{\circ} \mathrm{C}$. Decorridas até $72 \mathrm{~h}$ contava-se o número de ovos eclodidos, revelando o número de ovos viáveis. Foi estabelecido um experimento bifatorial, com três tratamentos.níveis ${ }^{-1}$ correspondentes ao Fator $\mathbf{A}$ (temperaturas controladas de condicionamento de adultos $=5^{\circ} \mathrm{C}, 10^{\circ} \mathrm{C}, 25^{\circ} \mathrm{C} \pm 2^{\circ} \mathrm{C}$ ) e seis tratamentos. níveis ${ }^{-1}$ correspondentes ao Fator $\mathbf{B}$ (tempo de exposição de adultos em B.O.D. = sete, 14, 21, 28, 35 e 42 dias).

O delineamento experimental foi inteiramente casualizado, com três repetições estatísticas, e os resultados obtidos a partir das variáveis respostas analisadas [sobrevivência total de adultos (fêmeas + machos), sobrevivência de fêmeas, sobrevivência de machos, longevidade de adultos, número/médio de ovos. fêmeas ${ }^{-1}$ e viabilidade (\%) de ovos], foram submetidos à análise de variância (Teste F) através do software
"WinStat", de acordo com Machado e Conceição (2002), para verificar a interação entre os fatores A e B.

Para as médias dos resultados obtidos entre os níveis do Fator A, para cada nível do Fator B, para as variáveis respostas, foi realizado teste de comparação de médias, com a finalidade de observar se cada temperatura controlada de condicionamento de adultos era limitante dentro de cada tempo de exposição de adultos em B.O.D.

\section{Resultados e Discussão}

Procedendo-se a análise dos resultados referentes à variável resposta sobrevivência de adultos de $M$. domestica (fêmeas + machos, fêmeas e machos) mantidos a $5^{\circ} \mathrm{C}$, verificou-se que houve sobrevivência apenas na exposição por sete dias $(27,00),(14,33)$ e $(12,67)$ respectivamente, porém não diferindo dos resultados obtidos a partir dos demais tratamentos. Para a temperatura de $10^{\circ} \mathrm{C}$, observou-se a ocorrência de sobreviventes (fêmeas + machos e fêmeas) nos períodos de sete $(24,33)(14,33), 14(17,67)(12,33)$ e $21(13,33)$ $(9,67)$ dias, respectivamente, que diferiram do controle apenas aos 21 dias. Já para os machos foi verificada a ocorrência de diferença entre a temperatura de $25^{\circ} \mathrm{C} \pm 2^{\circ} \mathrm{C}$ $(8,33)$ com as demais temperaturas, apenas aos 14 dias de exposição (Tabela 1).

Observou-se que a $25^{\circ} \mathrm{C} \pm 2^{\circ} \mathrm{C}$ a ocorrência de sobreviventes (fêmeas + machos, fêmeas e machos) estendeu-se até 28 dias $(2),(0,67)$ e $(1,33)$ respectivamente. Entretanto os resultados referentes a este período não

TABELA 1: Sobrevivência de Musca domestica (fêmeas + machos, fêmeas e machos) expostos as temperaturas constantes de $5^{\circ} \mathrm{C}, 10^{\circ} \mathrm{C}$ e $25^{\circ} \mathrm{C}+2^{\circ} \mathrm{C}$, em laboratório. Médias acompanhadas por letras distintas nas linhas diferem entre si através do teste de Tukey ( $5 \%$ de probabilidade).

\begin{tabular}{|c|c|c|c|c|c|c|c|c|c|}
\hline \multirow{2}{*}{$\begin{array}{l}\text { Exposição } \\
\text { (dias) }\end{array}$} & \multicolumn{3}{|c|}{$\begin{array}{c}\text { Sobrevivência } \\
\left(Q+0^{\lambda}\right)\end{array}$} & \multicolumn{3}{|c|}{$\begin{array}{l}\text { Sobrevivência } \\
()\end{array}$} & \multicolumn{3}{|c|}{$\begin{array}{c}\text { Sobrevivência } \\
(đ)\end{array}$} \\
\hline & $5^{\circ} \mathrm{C}$ & $10^{\circ} \mathrm{C}$ & $25^{\circ} \mathrm{C}$ & $5^{\circ} \mathrm{C}$ & $10^{\circ} \mathrm{C}$ & $25^{\circ} \mathrm{C}$ & $5^{\circ} \mathrm{C}$ & $10^{\circ} \mathrm{C}$ & $25^{\circ} \mathrm{C}$ \\
\hline 7 & $27^{a}$ & $24,33^{a}$ & $23,67^{a}$ & $14,33^{a}$ & $14,33^{\mathrm{a}}$ & $11,67^{\mathrm{a}}$ & $12,67^{a}$ & $10^{\mathrm{a}}$ & $12^{\mathrm{a}}$ \\
\hline 14 & $0^{\mathrm{b}}$ & $17,67^{\mathrm{a}}$ & $12,67^{\mathrm{a}}$ & $0^{\mathrm{a}}$ & $12,33^{\mathrm{a}}$ & $9,33^{\mathrm{a}}$ & $0^{\mathrm{b}}$ & $5,33^{\mathrm{b}}$ & $8,33^{\mathrm{a}}$ \\
\hline 21 & $0^{\mathrm{b}}$ & $13,33^{\mathrm{a}}$ & $6^{\mathrm{b}}$ & $0^{c}$ & $9,67^{\mathrm{a}}$ & $2,33^{b}$ & $0^{\mathrm{a}}$ & $3,67^{\mathrm{a}}$ & $3,67^{\mathrm{a}}$ \\
\hline 28 & $0^{\mathrm{a}}$ & $0^{\mathrm{a}}$ & $2^{\mathrm{a}}$ & $0^{\mathrm{a}}$ & $0^{\mathrm{a}}$ & $0,67^{\mathrm{a}}$ & $0^{\mathrm{a}}$ & $0^{\mathrm{a}}$ & $1,33^{\mathrm{a}}$ \\
\hline 35 & $0^{\mathrm{a}}$ & $0^{\mathrm{a}}$ & $0^{\mathrm{a}}$ & $0^{\mathrm{a}}$ & $0^{\mathrm{a}}$ & $0^{\mathrm{a}}$ & $0^{\mathrm{a}}$ & $0^{\mathrm{a}}$ & $0^{\mathrm{a}}$ \\
\hline 42 & $0^{\mathrm{a}}$ & $0^{\mathrm{a}}$ & $0^{\mathrm{a}}$ & $0^{\mathrm{a}}$ & $0^{\mathrm{a}}$ & $0^{\mathrm{a}}$ & $0^{\mathrm{a}}$ & $0^{\mathrm{a}}$ & $0^{\mathrm{a}}$ \\
\hline
\end{tabular}


diferem dos resultados obtidos das demais temperaturas (Tabela 1).

De acordo com Silveira-Neto (1976), Dajoz (1983), Wolda (1988), Torres et al. (2002) e Almeida e Gonçalves (2007), os resultados deste experimento somam-se aos conhecimentos de que os insetos são de natureza exotérmica e os processos fisiológicos de $M$. domestica referentes à sobrevivência foram condicionados pela sensibilidade às baixas temperaturas $5^{\circ} \mathrm{Ce} 10^{\circ} \mathrm{C}$, as quais interferiram sobre o desenvolvimento da população de insetos durante a estocagem.

Estes resultados estão referendados por Martins e Barbeitos (2000), onde enfatizam que muitas adaptações fisiológicas e comportamentais estão condicionadas a detecção de sinais resultantes de variações ambientais, determinantes da transição para uma nova situação ambiental, indicativo de variação no grau de adequação ao meio.

Como estes adultos sob confinamento a baixas temperaturas não apresentaram a oportunidade de se dispersar ou procurar condições climáticas mais adequadas ao seu desenvolvimento, acabaram sofrendo a interferência das adversidades climáticas, sendo confirmado por Minkin e Scott (1960) que, sob temperaturas inferiores a $10^{\circ} \mathrm{C}$, moscas adultas em geral, reduzem seu ciclo vital. Somando-se a citação de Torres et al. (2002), os quais postularam que as variações de temperatura influenciam sobre o tempo de duração de seu ciclo vital e o tamanho populacional.

Para estudo da longevidade de $M$. domestica (fêmeas + machos, fêmeas e machos) sob temperatura controlada $25^{\circ} \mathrm{C} \pm 2^{\circ} \mathrm{C}$ (controle), verificou-se que foi de (31), (30) e (29) dias, respectivamente. Já para a estocagem de adultos (fêmeas + machos, fêmeas e machos) a $5^{\circ} \mathrm{C}$, garantiu o estudo da longevidade apenas por sete dias de exposição em B.O.D. (33), $(29,67)$ e $(28,67)$ dias, respectivamente, causando uma interrupção na atividade biológica, porém não diferindo das demais temperaturas. Entretanto, sob temperatura de $10^{\circ} \mathrm{C}$, para (fêmeas + machos, fêmeas e machos), observou-se que a ocorrência de sobreviventes extendeu-se até 21 dias $(31,33),(25)$ e (24) dias, respectivamente (Tabela 2 ).

Desta maneira pode-se inferir que a utilização de baixas temperaturas $\left(5^{\circ} \mathrm{C}\right.$ e $\left.10^{\circ} \mathrm{C}\right)$, como forma de reduzir as taxas metabólicas e o desenvolvimento destes insetos na forma adulta foi uma estratégia de estocagem que interferiu sobre a longevidade (fêmeas + machos) de M. domestica.

Justus (2002), estudando a morfologia, assimetria flutuante e tabelas de vida e de fertilidade em $M$. domestica, conclui através da análise de curvas de sobrevivência que fêmeas apresentam maior longevidade quando comparadas aos machos, e que moscas isoladas (ausência de cópula) apresentam longevidade e expectativa de vida maiores do que as moscas agrupadas (presença de sexo), indicando que o sexo pode ser um fator limitante na longevidade.

Para estudo do número médio de ovos.fêmeas ${ }^{-1} \mathrm{a}$ temperatura controlada de condicionamento $25^{\circ} \mathrm{C} \pm 2^{\circ} \mathrm{C}$, verificou-se que o resultado foi de 86,69 ovos. fêmeas ${ }^{-1}$. Já a temperatura de $5^{\circ} \mathrm{C}$, garantiu o estudo da ovipostura apenas para fêmeas sob exposição por

TABELA 2: Longevidade de Musca domestica (fêmeas + machos, fêmeas e machos) expostos as temperaturas constantes de $5^{\circ} \mathrm{C}, 10^{\circ} \mathrm{C}$ e $25^{\circ} \mathrm{C}+2^{\circ} \mathrm{C}$, em laboratório. Médias acompanhadas por letras distintas nas linhas diferem entre si através do teste de Tukey ( $5 \%$ de probabilidade).

\begin{tabular}{c|c|c|c|c|c|c|c|c|c}
\hline \multirow{2}{*}{$\begin{array}{c}\text { Exposição } \\
\text { (dias) }\end{array}$} & \multicolumn{3}{|c|}{$\begin{array}{c}\text { Longevidade } \\
\left(0+0^{\lambda}\right)\end{array}$} & \multicolumn{3}{c|}{$\begin{array}{c}\text { Longevidade } \\
(+)\end{array}$} & \multicolumn{3}{c}{$\begin{array}{c}\text { Longevidade } \\
\left({ }^{\lambda}\right)\end{array}$} \\
\cline { 2 - 10 } & $5^{\circ} \mathrm{C}$ & $10^{\circ} \mathrm{C}$ & $25^{\circ} \mathrm{C}$ & $5^{\circ} \mathrm{C}$ & $10^{\circ} \mathrm{C}$ & $25^{\circ} \mathrm{C}$ & $5^{\circ} \mathrm{C}$ & $10^{\circ} \mathrm{C}$ & $25^{\circ} \mathrm{C}$ \\
\hline 7 & $33^{\mathrm{a}}$ & $28,33^{\mathrm{a}}$ & 31 & $29,67^{\mathrm{a}}$ & $27,67^{\mathrm{a}}$ & 30 & $28,67^{\mathrm{a}}$ & $23,33^{\mathrm{a}}$ & 29 \\
\hline 14 & $0^{\mathrm{b}}$ & $31,33^{\mathrm{a}}$ & - & $0^{\mathrm{b}}$ & $30^{\mathrm{a}}$ & - & $0^{\mathrm{b}}$ & $21^{\mathrm{a}}$ & - \\
\hline 21 & $0^{\mathrm{b}}$ & $31,33^{\mathrm{a}}$ & - & $0^{\mathrm{b}}$ & $25^{\mathrm{a}}$ & - & $0^{\mathrm{b}}$ & $24^{\mathrm{a}}$ & - \\
\hline 28 & $0^{\mathrm{a}}$ & $0^{\mathrm{a}}$ & - & $0^{\mathrm{a}}$ & $0^{\mathrm{a}}$ & - & $0^{\mathrm{a}}$ & $0^{\mathrm{a}}$ & - \\
\hline 35 & $0^{\mathrm{a}}$ & $0^{\mathrm{a}}$ & - & $0^{\mathrm{a}}$ & $0^{\mathrm{a}}$ & - & $0^{\mathrm{a}}$ & $0^{\mathrm{a}}$ & - \\
\hline 42 & $0^{\mathrm{a}}$ & $0^{\mathrm{a}}$ & - & $0^{\mathrm{a}}$ & $0^{\mathrm{a}}$ & - & $0^{\mathrm{a}}$ & $0^{\mathrm{a}}$ & - \\
\hline
\end{tabular}


sete dias $(25,14)$. Entretanto sob temperatura de $10^{\circ} \mathrm{C}$, observou-se ovipostura nos períodos de sete, 14 e 21 dias de exposição em B.O.D. com respectivamente $(51,58)$, $(24,33)$ e $(20,91)$ ovos.fêmeas $^{-1}$, valores numericamente inferiores ao tratamento controle $(86,69)$ (Tabela 3 ).

Fletcher et al. (1990) verificaram que para $M$. domestica há diminuição da fecundidade com a elevação da temperatura, devido ao menor tempo de vida, enquanto os extremos de temperatura afetam mais rapidamente a reprodução das fêmeas do que outras funções fisiológicas.

Para estudo da viabilidade de ovos de $M$. domestica a temperatura controlada de $25^{\circ} \mathrm{C} \pm 2^{\circ} \mathrm{C}$, foi de $86,74 \%$. Já na temperatura de $5^{\circ} \mathrm{C}$, foi obtida apenas para fêmeas sob exposição por sete dias $(72,12 \%)$, porém não diferindo dos dados referentes ao tratamento $10^{\circ} \mathrm{C}(81,92 \%)$ e semelhante aos resultados obtidos para o tratamento controle (86,74\%) (Tabela 3).
Entretanto a $10^{\circ} \mathrm{C}$, observou-se viabilidade referente aos períodos de exposição de sete $(81,92 \%), 14$ $(73,55 \%)$ e 21 dias $(64,95 \%)$, diferindo dos resultados obtidos sob temperatura de $5^{\circ} \mathrm{C}$ aos $14(0 \%)$ e 21 dias $(0 \%)$ cuja viabilidade de ovos foi inversamente proporcional ao tempo de exposição (Tabela 3 ).

Sob as condições experimentais e de acordo com o objetivo proposto, pode-se concluir que:

a) $\mathrm{O}$ procedimento de estocagem sob a influência de baixas temperaturas controladas de condicionamento $\left(5^{\circ} \mathrm{C}\right.$ e $\left.10^{\circ} \mathrm{C}\right)$, é uma estratégia que interfere negativamente sobre a sobrevivência de adultos (fêmeas + machos), sobrevivência de fêmeas, sobrevivência de machos, longevidade, número médio de ovos.fêmeas ${ }^{-1}$ e viabilidade de ovos de $M$. domestica quando comparadas a temperatura de $25^{\circ} \mathrm{C} \pm 2^{\circ} \mathrm{C}$;

b) $\mathrm{O}$ tempo de exposição de adultos de $M$. domestica em B.O.D. por sete dias, permite a ocorrência de sobrevivência total (fêmeas + machos), sobre-

TABELA 3: Número médio de ovos.fêmeas ${ }^{-1}$ e viabilidade (\%) de ovos obtidos a partir de fêmeas de Musca domestica expostas as temperaturas constantes de $5^{\circ} \mathrm{C}, 10^{\circ} \mathrm{C}$ e $25^{\circ} \mathrm{C}+2^{\circ} \mathrm{C}$, em laboratório. Médias acompanhadas por letras distintas nas linhas diferem entre si através do teste de Tukey (5\% de probabilidade).

\begin{tabular}{c|c|c|c|c|c|c}
\hline \multirow{2}{*}{$\begin{array}{c}\text { Exposição } \\
\text { (dias) }\end{array}$} & \multicolumn{3}{|c|}{$\begin{array}{c}\text { No médio de } \\
\text { ovos.fêmeas }\end{array}$} & \multicolumn{3}{c}{$\begin{array}{c}\text { Viabilidade } \\
\text { (\%) de ovos }\end{array}$} \\
\cline { 2 - 7 } & $5^{\circ} \mathrm{C}$ & $10^{\circ} \mathrm{C}$ & $25^{\circ} \mathrm{C}$ & $5^{\circ} \mathrm{C}$ & $10^{\circ} \mathrm{C}$ & $25^{\circ} \mathrm{C}$ \\
\hline 7 & $25,14^{\mathrm{a}}$ & $51,58^{\mathrm{a}}$ & 86,69 & $72,12^{\mathrm{a}}$ & $81,92^{\mathrm{a}}$ & 86,74 \\
\hline 14 & $0^{\mathrm{a}}$ & $24,33^{\mathrm{a}}$ & - & $0^{\mathrm{b}}$ & $73,55^{\mathrm{a}}$ & - \\
\hline 21 & $0^{\mathrm{b}}$ & $20,91^{\mathrm{a}}$ & - & $0^{\mathrm{b}}$ & $64,95^{\mathrm{a}}$ & - \\
\hline 28 & $0^{\mathrm{a}}$ & $0^{\mathrm{a}}$ & - & $0^{\mathrm{a}}$ & $0^{\mathrm{a}}$ & - \\
\hline 35 & $0^{\mathrm{a}}$ & $0^{\mathrm{a}}$ & - & $0^{\mathrm{a}}$ & $0^{\mathrm{a}}$ & - \\
\hline 42 & $0^{\mathrm{a}}$ & $0^{\mathrm{a}}$ & - & $0^{\mathrm{a}}$ & $0^{\mathrm{a}}$ & - \\
\hline
\end{tabular}


vivência de fêmeas, sobrevivência de machos, longevidade; um maior número médio de ovos. fêmeas $^{-1}$ e viabilidade de ovos.

\section{Referências}

ALMEIDA, F. S.; GONÇALVES, L. Efeitos da temperatura e do alimento no desenvolvimento de Dysdercus maurus Distant (Hemiptera, Pyrrhocoridae). Revista Brasileira de Entomologia, Curitiba, v. 51, n. 4, p. 506-511, 2007.

BERRYMAN, A. A. Population cycles: the case for trophic interactions. Oxford. 2002. Disponível em: <http://books.google. com.br/books?id=OxGmC- 5uaGMC\&pg=PA177\&lpg=PA177\&d q=Population+cycles:+The+case+for+trophic+interactions.\&sourc $\mathrm{e}=$ bl\&ots $=-$ WTQlnVOye\&sig $=\mathrm{j} 4 \mathrm{whLjUCDFmUnHX3L8OFGeiy}$ kds\&hl=pt-BR\&ei $=$ HsTtfzIYGEtgf5oPzaBQ\&sa $=X \&$ oi $=$ book_re sult\&ct $=$ result\&resnum $=6 \&$ ved $=0$ CFAQ6AEwBQ $\# v=$ onepage $\& q$ $\& \mathrm{f}=$ false $>$. Acesso em: 11 jul. 2011.

BICHÃO, M. H. C. F. Biotecnologia de produção de Chrysoperla cárnea (Stephens) (Neuroptera, Chrysopidae) e sua aplicação em controle biológico. 1989. 145 f. Relatório (Estágio de Licenciatura em Recursos Faunísticos e Ambiente) - Universidade de Évora, Évora. 1989.

DAJOZ, R. Ecologia Geral. Petrópolis: Vozes, 1983. 472 p.

FERREIRA, M. J. M.; LACERDA, P. V. Muscóides sinantrópicos associados ao lixo urbano em Goiânia, Goiás. Revista Brasileira de Zoologia, Curitiba, v. 10, n. 2, p. 185-195, 1993.

FLETCHER, M. G.; AXTELL, R. C.; STINNER, R. E. Longevity and fecundity of Musca domestica (Diptera, Muscidae) as a function of temperature. Journal of Medical Entomology, Raleigh, v. 27, n. 5, p. 922-926, 1990.

JUSTUS, A. Morfometria, assimetria flutuante e tabelas de vida e de fertilidade em Musca domestica L. (Diptera, Muscidae). 2002. 102 f. Dissertação (Mestrado em Parasitologia) - Universidade Estadual de Campinas, Campinas. 2002.

LARA, F. M. Princípios de Entomologia. São Paulo: Ícone, 1995. $331 \mathrm{p}$.

MACEDO, L. P. M. Desenvolvimento, reprodução e comportamento de Chrysoperla externa (Hagen, 1861) (Neuroptera, Chrysopidae) em diferentes condições ambientais. 2001. 78 f. Dissertação (Mestrado em Agronomia) - Universidade Federal de Lavras, Lavras. 2001.
MACHADO, A.; CONCEIÇÃO, A. R. Programa estatístico WinStat - Sistema de Análise Estatística para Windows, versão 2.0. Pelotas: UFPEL. 2002.

MARTINS, A. L.; NUNES, J. F.; ZAMPIERON, S. L. M. Levantamento da himenopterofauna (Classe Insecta) em uma mata de galeria contida numa matriz de pasto, no município de Pratápolis (MG), através da armadilha de Möericke. Ciência et Praxis, Passos, v. 3, n. 5, p. 7-12, 2010.

MARTINS, R. P.; BARBEITOS, M. S. Adaptações de insetos a mudanças no ambiente: ecologia e evolução da diapausa. In: MARTINS, R. P.; LEWINSOHN, T. M; BARBEITOS, M. S. (Ed.). Ecologia e comportamento de insetos. Vol. VIII. Série Oecologia Brasiliensis, Rio de Janeiro: UFRJ. 2000. p. 149-192.

MINKIN, J. S. E.; SCOTT, H. G. House fly pupation under baseboards. Journal of Economic Entomology, Riverside, v. 53, p. 479-480, 1960.

ODUM, E. P. Ecologia. Rio de Janeiro: Guanabara Koogan, 1988. $434 \mathrm{p}$.

PARRA, J. R. P. Técnicas de criação de Anagasta kuehniella, hospedeiro alternativo para produção de Trichogramma. In: PARRA, J. R. P.; ZUCCHI, R. A. (Ed.). Trichogramma e o controle biológico aplicado. Piracicaba: FEALQ, 1997. 324 p.

PARRA, J. R. P. Técnicas de criação de insetos para programas de controle biológico. Piracicaba: ESALQ/FEALQ, 2001. 134 p.

PEGG, D. E.; M. P. DIAPER. On the mechanism of injury to slowly frozen erythrocytes. Biophysical Journal, Cambridge, v. 54, p. 471-488, 1988.

RODRIGUES, W. C. Fatores que influenciam no desenvolvimento dos insetos. Info Insetos, 2004. Disponível em: <http://www. infoinsetos.ebras.bio.br/pdf/art0104-01.pdf>. Acesso em: 22 ago. 2011.

SANTOS, I. R. I. Criopreservação: potencial e perspectivas para a conservação de germoplasma vegetal. Revista Brasileira de Fisiologia Vegetal, Campinas, v. 12, p. 70-84, 2000.

SILVEIRA-NETO, S. Manual de Ecologia dos Insetos. São Paulo: Agronômica Ceres, 1976. 419 p.

TORRES, J. R.; OLIVEIRA, C. M. B.; WALD, V. B. Influência sazonal sobre os períodos de pré-pupa e de pupa de Musca domestica, na região de Porto Alegre, RS, Brasil. Acta Scientiae Veterinariae, Porto Alegre, v. 30, p. 37-42, 2002.

WOLDA, H. Insect seasonality: why? Annual Review of Ecology and Systematics, Palo Alto, n. 19, p. 1-18, 1988. 